\title{
Visualization of Magnetic Surfaces during Current Ramp-Up Phase Using Thermal Lithium Sheet Beam in CPD
}

\author{
Tetsushi KIKUKAWA, Hideki ZUSHI ${ }^{1)}$, Tomohiro MORISAKI ${ }^{2}$, \\ Rajendraprasad BHATTACHARYAY, Tomofumi RYOUKAI, Tomokazu YOSHINAGA ${ }^{1)}$, \\ Makoto HASEGAWA $^{1)}$, Kazuaki HANADA ${ }^{1)}$, Hiroshi IDEI ${ }^{1)}$ and CPD Experimental Group ${ }^{1)}$ \\ Interdisciplinary Graduate School of Engineering Science, Kyushu University, Kasuga, Fukuoka 816-8580, Japan \\ 1) Research Institute for Applied Mechanics, Kyushu University, Kasuga, Fukuoka 816-8580, Japan \\ 2) National Institute for Fusion Science, Toki, Gifu, 509-8292, Japan
}

(Received 11 January 2008 / Accepted 21 January 2008)

\begin{abstract}
Two dimensional electron density profile measurement has been performed in the spherical tokamak CPD (Compact PWI experimental Device) using Li sheet beam imaging technique. The topological change from the open magnetic field line configuration to the closed one is visualized by this technique. The plasma current can be driven by RF waves itself in a weak mirror configuration and a clear change is observed in plasma boundary as well as magnetic field topology associated with the transition of the current from low $(\sim 1 \mathrm{kA})$ to high $(\sim 3 \mathrm{kA})$ value.
\end{abstract}

(c) 2008 The Japan Society of Plasma Science and Nuclear Fusion Research

Keywords: Li beam, electron density profile, ECRH, EBW, current drive, magnetic flux surface

DOI: $10.1585 /$ prr.3.010

In CDX-U, DIII-D and LATE the electron cyclotron waves have been used to ramp-up the plasma current under open field configuration with the combination of the toroidal and vertical magnetic field lines [1,2]. However, the physics of current ramp-up mechanism, that is, magnetic reconnection, is still not clear. In CPD the sheet thermal Li beam and CCD imaging method have been used to get two dimensional density profile in the wide area [3-5]. This paper describes the first result of visualization of the topological change in magnetic configuration during the RF driven plasma current ramp-up by imaging technique.

CPD is a spherical tokamak device whose diameter as well as height is $\sim 1.2 \mathrm{~m}$ as shown in Fig. 1. The diameter of the center stack is $0.255 \mathrm{~m}$. The working gas is hydrogen and typical fuelling pressure is $\sim 1.0 \times 10^{-5}$ Torr. Four toroidal coils produce the magnetic field of $0.29 \mathrm{~T}$ at the major radius $R \sim 0.19 \mathrm{~m}$. Three sets of poloidal field (PF) coils are used to create mirror configurations (decay index $\sim 0.046, B_{\mathrm{Z}} \sim 40 \mathrm{G}$ at $R=0.2 \mathrm{~m}$ ). In these experiments $1.5-60 \mathrm{~kW}$ of RF power $(8.2 \mathrm{GHz})$ is injected from the lower field side of the CPD to initiate and sustain the plasma. The RF driven plasma current is measured with Rogowski coils installed inside the CPD chamber.

The Li beam injector [3] is located at the bottom of the chamber and the beam is injected at an oblique angle of 18.18 degree with respect to the machine axis at $R \sim 0.29 \mathrm{~m}$ (see Fig. 1). The full width at half maximum of the sheet beam is $40 \mathrm{~mm}$ in the toroidal direction. The oven is heated up to a temperature of $\sim 900 \mathrm{~K}$. The image of

author's e-mail: kikukawa@triam.kyushu-u.ac.jp

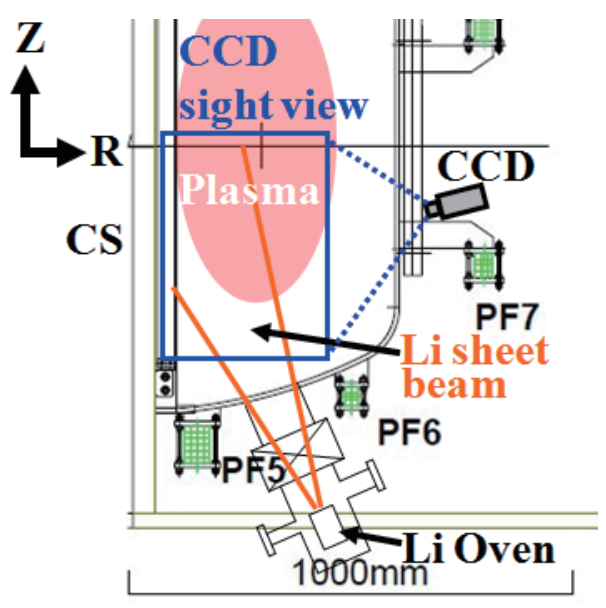

Fig. 1 Cross section of CPD and Li injector.

$\operatorname{LiI}(670.8 \mathrm{~nm})$ light intensity is detected with a CCD camera equipped with a lens and an optical filter whose FWHM is $5 \mathrm{~nm}$. The spatial and temporal resolutions are $1 \mathrm{~mm}$ and $1 \mathrm{~ms}$, respectively.

A vertically extended slab image is observed at the low RF power $(\sim 1.5 \mathrm{~kW})$ phase, which indicates that an initial plasma is formed along the 1st resonance layer [4]. Furthermore, this image is found to be convexly curved, which agrees with the contour of the poloidal magnetic flux surfaces $\Phi(R, Z)$ without plasma current.

Plasma current $\left(I_{\mathrm{p}}\right)$ starts to ramp-up with the ramping up of RF power $\left(P_{\mathrm{rf}}\right)$. It is found that a current transition occurs, when $P_{\mathrm{rf}}$ exceeds a critical value $(\sim 22 \mathrm{~kW})$, as shown in Fig. 2. The transition occurs within $2-3 \mathrm{~ms}$ and 


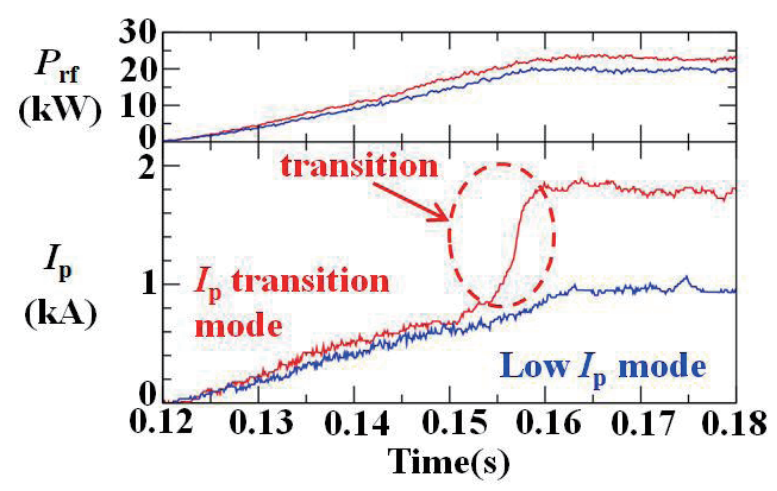

Fig. 2 Two types of discharges with and without $I_{\mathrm{p}}$ transition.
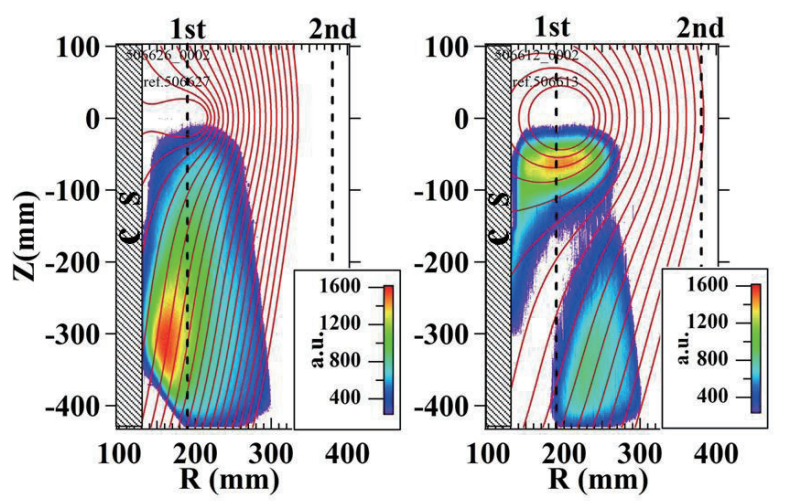

Fig. 3 Li image in (left) low $I_{\mathrm{p}}$ mode $\left(I_{\mathrm{p}} \sim 1 \mathrm{kA}\right)$ and (right) $I_{\mathrm{p}}$ transition mode $\left(I_{\mathrm{p}} \sim 2.5 \mathrm{kA}\right)$.

the current abruptly increases from $1 \mathrm{kA}$ (low $I_{\mathrm{p}}$ mode) to $2-3 \mathrm{kA}\left(I_{\mathrm{p}}\right.$ transition mode). Below the critical $P_{\mathrm{rf}}, I_{\mathrm{p}}$ is kept at a low level.

Drastic changes are observed in the LiI images with and without current transition (see Fig. 3). In low $I_{\mathrm{p}}$ mode (Fig. 3 (left)), a slab image extends towards the lower field side. The contours of the $\Phi(R, Z)$ are also superposed on the respective image of $\operatorname{LiI}(R, Z)$. The $\Phi(R, Z)$ is calculated assuming a ring plasma current of $1 \mathrm{kA}$ with its center at $R=0.2 \mathrm{~m}$ and $Z=0 \mathrm{~m}$. Although the image becomes more convexly curved due to $I_{\mathrm{p}}(\sim 1 \mathrm{kA})$, the image near the mid plane does not indicate appearance of closed flux surfaces over a large volume.

When $I_{\mathrm{p}}$ reaches to a critical value, the image splits into two (inner and outer) parts vertically at $R \sim 0.2 \mathrm{~m}$. The inner part shrinks towards the center stack, but the outer part remains unchanged. As shown in Fig. 3 (right), with the current transition a circular high intensity region appears near the mid plane, suggesting the establishment of the last closed flux surface LCFS via magnetic reconnection. With addition of $B_{\mathrm{z}}$ the uni-directional PfrirshSchlüter sheet current can flow along tilted magnetic field lines. However, the negative induced current, driven by negative electric field near the center stack, is also expected during the current ramp-up phase. They may play an important role to split the image. It is noted that a very low
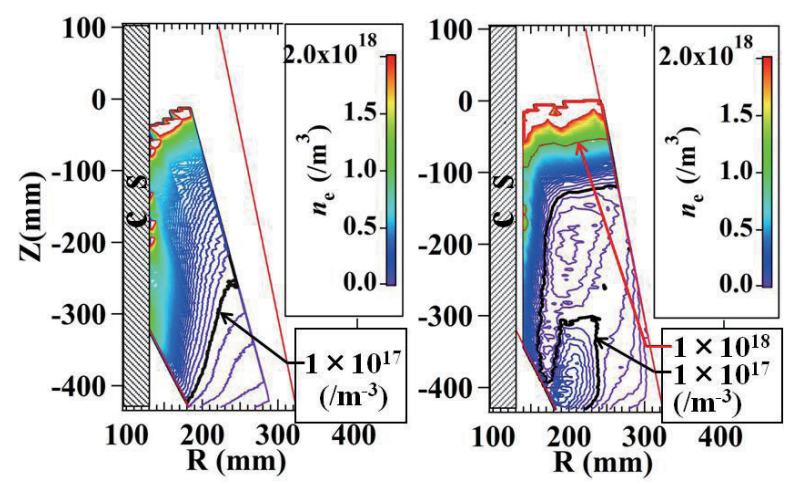

Fig. $4 n_{\mathrm{e}}(R, Z)$ in (left) low $I_{\mathrm{p}}$ mode and in (right) $I_{\mathrm{p}}$ transition mode.
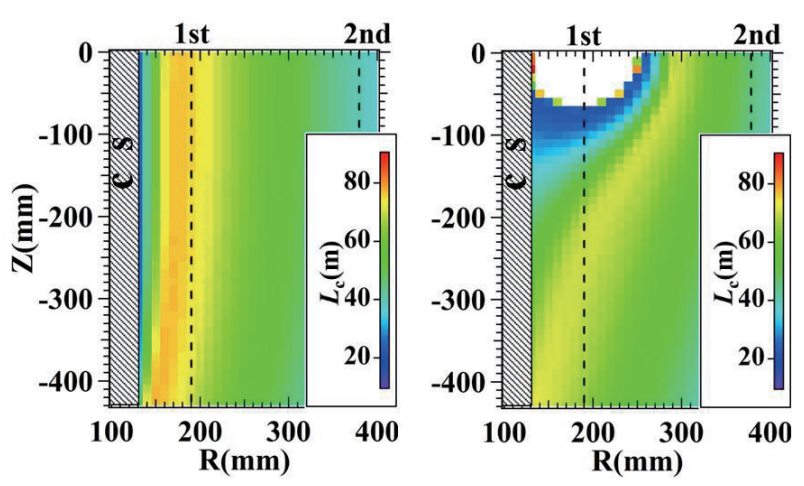

Fig. $5 L_{\mathrm{c}}$ contour at (left) $I_{\mathrm{p}}=0 \mathrm{kA}$ and (right) $I_{\mathrm{p}}=2.5 \mathrm{kA}$. These contours are symmetric with respect to mid plane $(Z=0)$

intensity region appears between circular and outer vertically "extended" images. Although 1 st resonance exists at $R=0.19 \mathrm{~m}$, observed very low intensity for $Z<-0.2 \mathrm{~m}$, suggesting a low density plasma, indicates that plasma is not produced at $R=0.19 \mathrm{~m}$. This region is, therefore, considered to be sustained by 2 nd harmonic coupling at $R=0.38 \mathrm{~m}$.

The electron density $n_{\mathrm{e}}(R, Z)$ contours for the above two cases are shown in Fig. 4 . In Fig. 4 (left) vertically extended equi-density contour follows well with $\Phi(R, Z)$. In Fig. 4 (right) a sharp density gradient near $Z \sim-0.1 \mathrm{~m}$ is found near the LCFS. Below the LCFS vertically extended low $n_{\mathrm{e}}(R, Z)$ with a depression region also follows the $\Phi(R, Z)$. Since the plasma cut off density is $7 \times 10^{17} \mathrm{~mm}^{-3}$, the over-dense plasma is produced by RF waves in the core region.

In order to understand the change in LiI images during the $I_{\mathrm{p}}$ ramp-up phase the contour of the wall-to-wall length $\left(L_{\mathrm{c}}\right)$ of the magnetic field lines are calculated for the above mentioned cases and shown in Fig. 5. For plasma to be observed $L_{\mathrm{c}}$ should exceed the ionization mean free path of electron $\left(\lambda_{\text {ion }}\right)$, which is estimated to be $\sim 40 \mathrm{~m}$. The maximum $(\sim 100 \mathrm{~m})$ of the $L_{\mathrm{c}}$ contour coincident with the resonance layer, therefore, suggests that a vertically extended plasma can be easily produced at $1.5 \mathrm{~kW}$ (Fig. 5 
(left)). The $L_{\mathrm{c}}$ contour is modified drastically when $I_{\mathrm{p}}$ transition occurs (Fig. 5 (right)). There is a region surrounding the closed magnetic surface, where $L_{\mathrm{c}}$ is shorter than $\lambda_{\text {ion }}$, which may contribute to the depressed density region. The remained longer $L_{\mathrm{c}}$ region strongly bent by $I_{\mathrm{p}}$, connecting with the 2 nd harmonic region, may also contribute to the outer part of vertically extended image from $Z=-0.4 \mathrm{~m}$ to $-0.3 \mathrm{~m}$. Although the trapped particle orbits are studied, there are still discrepancies to explain the exact position of depressed density region. This has been left for future.
This work was performed with the support and under the auspices of the NIFS Collaboration Research Program (NIFS07KOAR009, NIFS05KUTR012).

[1] C.B. Forest et al., Phys. Plasmas 1, 1568 (1994).

[2] T. Maekawa et al., Nucl. Fusion 45, 1439 (2005).

[3] H. Zushi et al., J. Nucl. Mater. 363, 1429 (2007).

[4] R. Bhattacharyay et al., Plasma Fusion Res. 2, S1103 (2007).

[5] R. Bhattacharyay et al., accepted for publication in Phys. Plasmas (2008). 